\title{
Role of the Dynamic Contact Angle on Splashing
}

\author{
Miguel A. Quetzeri-Santiago, ${ }^{1}$ Kensuke Yokoi, ${ }^{2}$ Alfonso A. Castrejón-Pita, ${ }^{3}$ and J. Rafael Castrejón-Pita ${ }^{1}$ \\ ${ }^{1}$ School of Engineering and Materials Science, Queen Mary, University of London, London E1 4NS, United Kingdom \\ ${ }^{2}$ School of Engineering, Cardiff University, Queen's Buildings, The Parade, Cardiff CF24 3AA, United Kingdom \\ ${ }^{3}$ Department of Engineering Science, University of Oxford, Parks Road, Oxford OX1 3PJ, United Kingdom
}

(Received 6 November 2018; published 5 June 2019)

\begin{abstract}
In this Letter, we study the splashing behavior of droplets upon impact onto a variety of substrates with different wetting properties, ranging from hydrophilic to superhydrophobic surfaces. In particular, we study the effects of the dynamic contact angle on splashing. The experimental approach uses high-speed imaging and image analysis to recover the apparent contact angle as a function of the spreading speed. Our results show that neither the Capillary number nor the so-called splashing parameter are appropriate to characterize the splashing behavior under these circumstances. However, we show that the maximum dynamic advancing contact angle and the splashing ratio $\beta$ adequately characterize the splashing behavior.
\end{abstract}

DOI: 10.1103/PhysRevLett.122.228001

A drop impacting onto a solid dry substrate can, among other several results, splash or spread over the solid surface. The result depends not only on the droplet properties and speed, but on a wide range of parameters including the atmospheric pressure and the surface roughness, surface microstructure, temperature, stiffness, and substrate speed [1-6]. The dynamics of drops is often characterized by the Weber $\left(\mathrm{We}=\left(\rho U^{2} D / \sigma\right)\right.$ ) and the Reynolds numbers $(\operatorname{Re}=(\rho U D / \mu))$, where $\rho, \sigma$, and $\mu$ are the liquid density, surface tension, and viscosity, respectively, $D$ is the drop diameter and $U$ is the impact velocity. At high $\mathrm{We}$ and $\mathrm{Re}$ numbers, an impacting drop ejects a thin film, which, in turn, breaks up to form secondary droplets; this phenomena is known as splashing [1].

Although many studies have aimed at finding scaling arguments to characterize splashing [7-13], the exact combination of parameters and their influence have remained elusive. In 2013 Palacios et al. found that, at $\operatorname{Re}>1000$, viscosity promotes both splashing and the gliding of the lamella [13], but small Re numbers inhibit the break up of the lamella, thus, reducing splashing $[13,14]$. Viscosity effects are not important for drops spreading and splashing over a thin sheet of air but are important if spreading occurs in contact with the surface $[15,16]$. Moreover, it has also been suggested that liquid viscosity has a nonmonotonic effect on splashing [17]. Other studies have revealed that the ambient pressure has a crucial role on spreading [18] and on splashing [19].

Published by the American Physical Society under the terms of the Creative Commons Attribution 4.0 International license. Further distribution of this work must maintain attribution to the author(s) and the published article's title, journal citation, and DOI.
Further studies have analyzed the role of the ambient gas on the lubrication force lifting the lamella [7,10,20,21] concluding that the surrounding gas viscosity is, arguably, the most influential parameter on splashing. Surprisingly, air at the impact point plays no significant role on splashing, but it is the air at the spreading edge that influences it $[8,22,23]$. Two widely used parameters in splashing are the splashing parameter $K=\mathrm{We}^{1 / 2} \mathrm{Re}^{1 / 4}[6,11,13]$, and the capillary number $\mathrm{Ca}=\mathrm{We} / \mathrm{Re}$ [24].

To date, there is no accepted consensus on the role of surface wettability on drop splashing. Surface wettability is often characterized by the static apparent contact angle $\theta_{s}$ formed by the intersection of the liquid-solid and the liquidvapor interfaces of a sessile droplet resting on a flat substrate. The interface where liquid, solid, and gas coexist is called the contact line. In a quasistatic condition, e.g., pumping liquid in or out of a sessile droplet, the static advancing $\theta_{\mathrm{sa}}$ and the static receding $\theta_{\text {sr }}$ contact angles can be defined [1]. Dynamic contact lines, found on liquids spreading over a solid, among other examples, define an advancing dynamic contact angle $\theta_{\mathrm{DA}}$, and a retracting contact line define a receding dynamic contact angle $\theta_{\mathrm{DR}}[25,26]$. Past studies have analyzed the influence of $\theta_{s}$ on splashing and determined that wettability has no effect on the splashing threshold for high capillary numbers [11,27], as the drop spreads over a thin air film and not along the substrate. A further study on rough substrates found no direct effect of wettability on splashing [11]. Contrasting studies have shown that the splashing depends on $\theta_{s}$ [14,28-31]. Experiments have shown that drop splashing effectively depends on the surface wettability $[14,30]$ and that hydrophobic substrates exhibit a low-velocity splashing threshold [30]. The influence of the dynamic contact angle $\left(\theta_{D}\right)$ on the splashing threshold has also been observed on simulations and found that splashing does not occur for $\theta_{\mathrm{DA}}<90^{\circ}$ [32]. 
In this Letter, a systematic study of liquid droplets impacting onto various solid substrates including glass, ultraclean mica, Glaco-coated surfaces, Perfluorodecyl acrylates (PFAC), and polytetrafluoroethylene is presented. In our experiments, the drop impact speed, the substrate wettability (hydrophilic, hydrophobic, and superhydrophobic), and the liquid properties were varied. Our results indicate that the splashing threshold depends on the dynamic contact angle and, therefore, is influenced by the wettability. Importantly, our experimental data indicate that smooth spreading, following impact, occurs at a contact angle of $\theta_{\mathrm{DA}} \geq 87^{\circ}$, including for substrates classified as hydrophilic by the traditionally used static contact angle convention. We show that the advancing dynamic contact angle, and the splashing ratio can effectively determine the splashing threshold.

Single liquid drops were generated by dripping, and allowed to travel vertically downwards towards dry solid substrates of different wetting properties. The drops were produced by a $1.0 \mathrm{~mm}$ diameter stainless steel needle attached to a syringe pump which provided the liquid at a rate of $1.94 \mathrm{~mm}^{3} / \mathrm{s}$ until the drop fell due to gravity. The distance from the needle to the substrate was varied from 0.4 to $2.0 \mathrm{~m}$, allowing us to adjust the impact speed $(U)$. The transition from spreading to splashing for all the liquidsubstrate combinations was then mapped by varying the impact speed. In these experiments, $U$ ranged from 1.1 to $4.9 \mathrm{~m} / \mathrm{s}$, and drop diameters ranged from $D=1.8$ to $2.5 \mathrm{~mm}$. All experiments were performed in air, at $\sim 25^{\circ} \mathrm{C}$ and at the regular atmospheric pressure. The dynamic apparent contact angle $\left(\theta_{D}\right)$ was measured for each liquidsolid substrate combination during the spreading and receding (if found) phases at an impact speed of $1.10 \mathrm{~m} / \mathrm{s}$.

Drop impacts were recorded by either a Phantom V710 or a Phantom V2512 high-speed camera coupled with a microscope lens and back illuminated by a diffuser-LED array in a traditional shadowgraph configuration. The resolution for spreading experiments was set to $1280 \times$ 256 pixels $^{2}$ at a sample rate of 23000 frames per second (fps), and an exposure of $10.0 \mu \mathrm{s}$. The effective resolution for spreading experiments is of $6.47 \mu \mathrm{m}$ per pixel. The effective resolution for splashing experiments was of $16.39 \mu \mathrm{m}$ per pixel at a resolution of $800 \times 128$ pixels $^{2}$, a sample rate of $83000 \mathrm{fps}$, and an exposure time of $1.0 \mu \mathrm{s}$. Figure 1 shows water and ethanol drops impacting on different substrates at various We numbers. Image analysis was performed on spreading experiments to extract $\theta_{D}$ at each frame by a custom MATLAB code. Figure 2 shows examples of these apparent dynamic contact angle measurements. In brief, the code works as follows: it takes an image and converts it to grey scale and then into a binary image using a set threshold. It then detects the boundary of the droplet, the substrate, and the contact point between the droplet and the substrate. At this point, the spreading diameter (distance between the two contact points) is

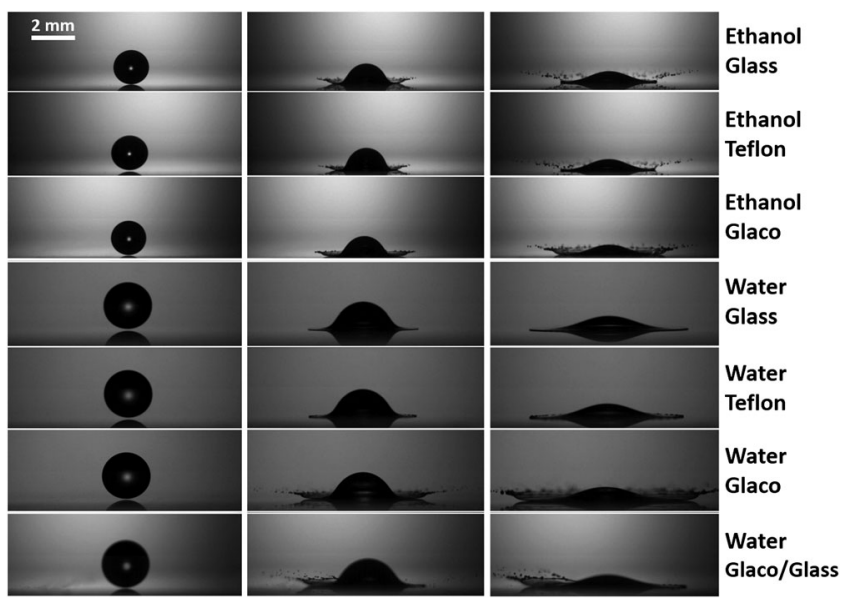

FIG. 1. Impact behavior for ethanol $(\mathrm{We}=571)$ and water $(\mathrm{We}=462)$ droplets on different substrates. Splashing is observed for ethanol on all substrates. In contrast, water presents splashing on Glaco, microsplashing for Teflon [33], and no splashing for glass. The bottom set of images shows the impact of a droplet on a glass substrate whose left side has been coated with Glaco; the left side of the droplet rapidly splashes while the right side spreads.

recorded to calculate the contact line speed $u_{\mathrm{CL}}$. The code then fits a second order polynomial with the least squares method to a a fraction of the droplet boundary near the contact line from which the apparent (dynamic) contact angle for each frame is computed at the pinning point. A complementary MATLAB code is used to extract the droplet diameter and impact velocity on both spreading and splashing experiments.

The working fluids were ethanol, tridistilled water, and an aqueous glycerol solution with a viscosity of $4.7 \mathrm{mPa}$. Eight different substrates were utilized and consisted of glass slides, ultraclean mica, cast acrylic, polytetrafluoroethylene(Teflon), Glaco-coated microscope slides, oxygenplasma treated Glaco-coated glass slides, $\mathrm{PFAC}_{6}$ and $\mathrm{PFAC}_{8}$ (Perfluorodecyl acrylates)-coated glass slides [34]. Only clean-fresh surfaces were used and experiments

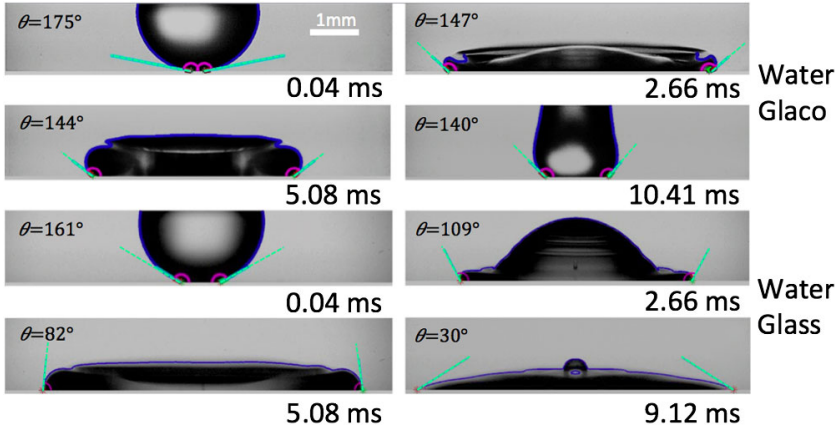

FIG. 2. Image analysis of water droplets impacting and spreading on glass and Glaco. The navy blue line represents the profile of the drop, the light blue line is the tangent to the droplet with respect to the pinning point. 


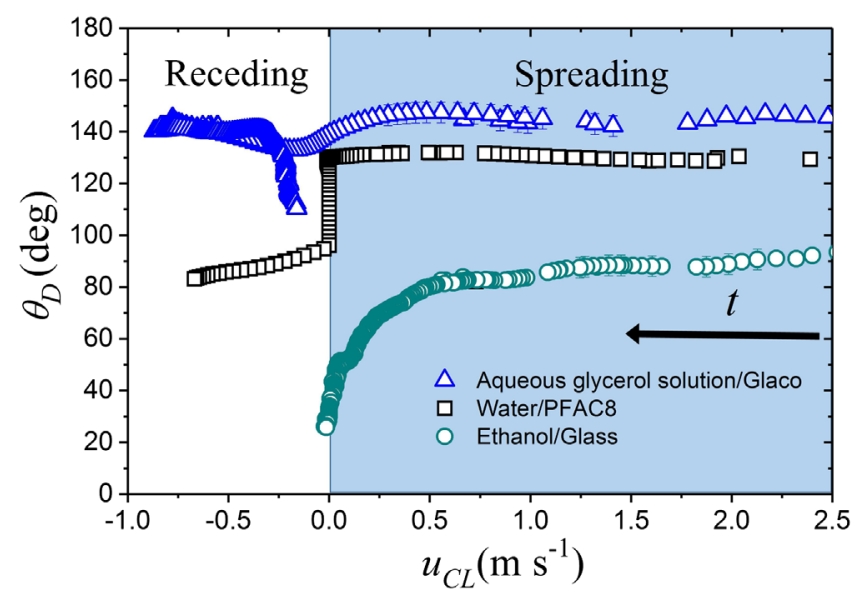

FIG. 3. The dynamic contact angle $\theta_{D}$ in terms of the contact line velocity. The three major wetting behaviors are seen, i.e., superhydrophobic (aqueous glycerol solution on Glaco), hydrophobic (water on $\mathrm{PFAC}_{8}$ ) and wetting (ethanol on glass). Here, $\theta_{\max }=147$ for glycerol and water on Glaco, $\theta_{\max }=131$ for water on $\mathrm{PFAC}_{8}$, and $\theta_{\max }=89$ for ethanol on glass. The impact velocity for water and the aqueous solution droplets is of $1.34 \mathrm{~m} / \mathrm{s}$, while for the ethanol drop, it is of $0.98 \mathrm{~m} / \mathrm{s}$.

were repeated a minimum of three times. The experiments covered a ranges of $130<\mathrm{We}<811,69<\mathrm{K}<299$, $0.027<\mathrm{Ca}<0.256$, and $\mathrm{Oh}=<0.011$, where the Ohnesorge number is $\mathrm{Oh}=(\mu / \sqrt{\rho \sigma D})$.

Figure 3 presents the dynamic contact angle in terms of the contact line speed $u_{\mathrm{CL}}$ for three liquid-substrate examples, and shows the three contrastingly different contact line dynamics, i.e., hydrophilic (ethanol on glass), hydrophobic (water on $\mathrm{PFAC}_{8}$ ), and superhydrophobic (aqueous glycerol solution on Glaco) dynamics. Here, for future analysis, we define $\theta_{\max }$ as the advancing asymptotic value of $\theta_{\mathrm{DA}}$, or that at a contact line speed of $2.0 \mathrm{~m} / \mathrm{s}$. At these timescales, there are no large surface deformations, and the contact angle can be measured precisely. As expected, no receding is observed under the hydrophilic condition as the contact line remains pinned at the maximum spreading diameter. An important observation from Fig. 4 is that the maximum dynamic contact angle is always $\geq 87^{\circ} \pm 4^{\circ}$, even for hydrophilic substrates. This indicates that, under most wetting conditions, impacting drops spread at an angle greater than their static contact angle. The extreme case is ethanol on glass with a static contact angle of $\theta_{s}=5^{\circ} \pm 4^{\circ}$ and a maximum advancing angle of $\theta_{\max }=87^{\circ} \pm 4^{\circ}$. In fact, two liquidsubstrate systems can have the same equilibrium contact angle $\left(\theta_{s}\right)$ but different dynamic contact angles. Ethanol on Glaco-surfaces, and ethanol on glass, present the same equilibrium contact angle, $\theta_{s}=5^{\circ} \pm 4^{\circ}$, yet their maximum advancing angles differ significantly, i.e., $\theta_{\max }=98^{\circ}$ and $87^{\circ}$, respectively. Similarly, water and the $4.7 \mathrm{mPa} \mathrm{s}$ solution have the same $\theta_{s}$ on glass, but their $\theta_{\max }$ differ by $10^{\circ}$ (larger for $4.7 \mathrm{mPas}$ solution). These results are in

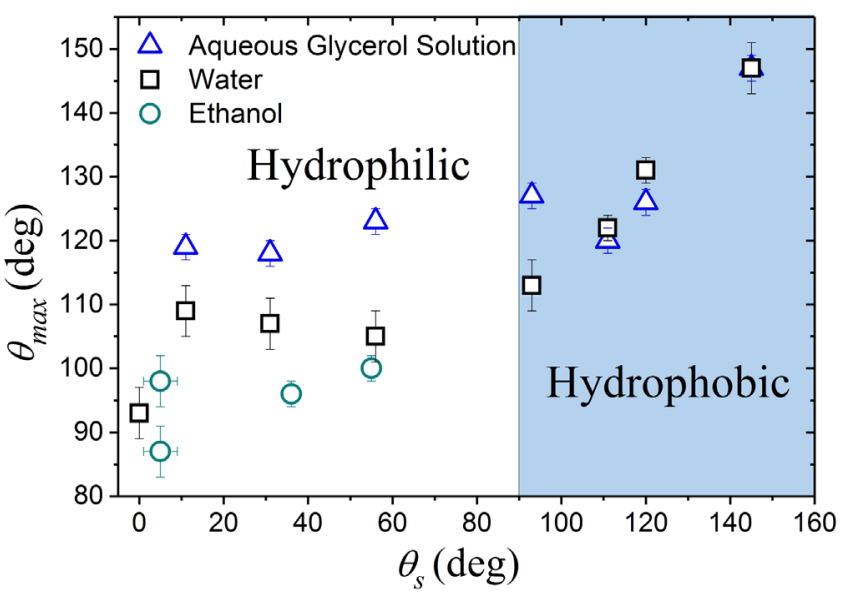

FIG. 4. The dynamic contact angle $\theta_{\max }$ in terms of the static contact angle $\theta_{s}$. For all of the liquids and substrates $\theta_{\max } \geq 87^{\circ}$ regardless $\theta_{s}$.

agreement with past reports arguing that the advancing contact angle is determined by both the droplet liquid surface tension and viscosity $[35,36]$. Our results also capture the contact angle hysteresis behavior for hydrophobic substrates $\left(\mathrm{PFAC}_{8}\right)$ found in previous papers [31], see Fig. 3. This hysteresis is observed at $u_{\mathrm{CL}}=0$ where the advancing and receding angles rapidly achieve their asymptotic values. The superhydrophobic (Glaco) substrate does not show a large contact angle variation, remaining at $\approx 140^{\circ}$ throughout the advancing and most of the receding phases, only to vary when the droplet is about to bounce off the substrate $\left(u_{\mathrm{CL}} \approx-0.25 \mathrm{~m} / \mathrm{s}\right)$. In agreement with past works, a low contact angle hysteresis is a requirement for superhydrophobic substrates [26,37]. Our experiments (example in the Supplemental Material [34]) and those by Goede et al. [21], confirm the lifting of the lamella and splashing near the critical impact velocity, occur in the range of 0.4 to $1.2 \mathrm{~ms}$ after impact. Consequently, we expect wettability and, hence, the contact angle to play an important role in this timescale (hundreds of microseconds). Accordingly, we found that the contact line speed is between 1.0 and $2.5 \mathrm{~m} / \mathrm{s}$, where the asymptotic maximum contact angle is found.

Splashing and no-splashing (spreading) events were visually identified from the experimental images. Here, splashing denotes an event in which the rim at the end of the ejecta breaks up to form at least one secondary droplet. Results indicate that splashing is favored by increasing impact speed and increasing maximum advancing contact angle $\theta_{\max }$. Figure 1 is critical to understanding the effects of wettability on splashing; i.e., it is easier to splash on surfaces with a larger maximum advancing contact angle. Ethanol drops impacting at $U=1.97 \mathrm{~m} / \mathrm{s} \quad(\mathrm{We}=258)$ show no splashing on the glass substrate $\left(\theta_{\max }=87\right)$, but splashing on the Glaco-covered substrate happens at $U=1.89 \mathrm{~m} / \mathrm{s}\left(\theta_{\max }=98\right)$, please see the Supplemental Material [34]. Water impacting onto Glaco-coated surfaces 

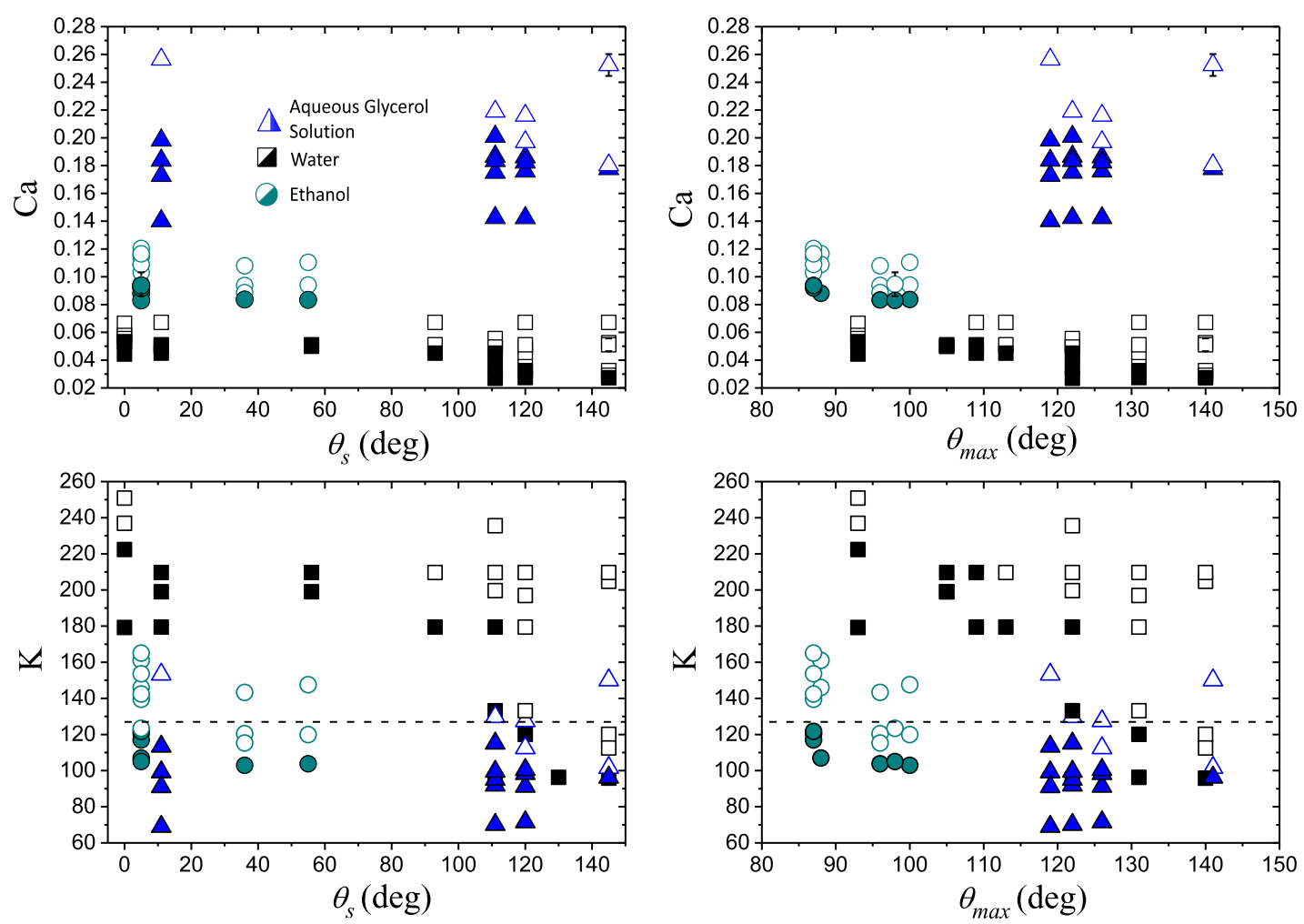

FIG. 5. Impact behavior in terms of the capillary $\mathrm{Ca}$ and the splashing $K$ parameter as a function of both the static $\theta_{s}$ and the maximum dynamic contact angles $\theta_{\max }$. Open symbols represent splashing and solid symbols no splashing. A good behavior divide "by liquid" is seen but is not consistent across all the fluids. The dotted line indicates the splashing threshold for ethanol drops, $K>127$, found by Bird et al. in 2009 [6].

at a speed of $U=2.09 \mathrm{~m} / \mathrm{s}\left(\mathrm{We}=167, \theta_{\max }=147\right)$ splashes, but when impacting $\mathrm{PFAC}_{8}$ at $U=2.34 \mathrm{~m} / \mathrm{s}$ $\left(\mathrm{We}=189, \theta_{\max }=131\right)$ no secondary drops are detached (fingering of the lamella but no splashing). These results are consistent with previous observations [14,30].

Traditionally, two dimensionless groups have been used to describe the splashing behavior; these are the capillary number, $\mathrm{Ca}$, and the splashing parameter, $K$, [12,24]. Accordingly, Fig. 5 presents the splashing behavior in terms of these groups and both the static contact angle $\left(\theta_{s}\right)$, and the maximum advancing contact angle $\left(\theta_{\max }\right)$. Previous experiments with ethanol drops impacting aluminium (wettable) placed the splashing threshold at $K=127$. As seen, our results are consistent with this finding as ethanol droplets splash on hydrophilic substrates at $K>120$ [6]. However, both groups ( $\mathrm{Ca}$ and $K$ ) fail to separate the overall splashing behavior for all the other liquids; the data are clustered by liquid, and within these clusters, the results show that large contact angles and high $\mathrm{Ca}$ numbers promote splashing. In particular, the critical $K=127$ for ethanol, found in [6], fails for the rest of the liquids. For water, the effect of wettability on splashing is progressively visible as the contact angle (static or dynamic) increases. An overall conclusion is that splashing, within clusters, is independent from the contact angle for hydrophilic substrates, i.e., $\theta_{s} \lesssim 90$ or $\theta_{\max } \lesssim 113^{\circ}$, and this evidence places our results in agreement with past works [27]. Recent works have described the splashing velocity of drops impacting smooth mostly hydrophilic surfaces at low Ohnesorge numbers, by numerically solving the momentum balance equation and estimating the aerodynamics lifting forces [7,21]. This splashing criteria, named the splashing ratio, incorporates the wedge angle, i.e., the angle between the lifted liquid sheet (ejecta) and the substrate at the moment of splashing, and the air viscosity. This is

$$
\beta \approx 2.22 \frac{1}{\tan (\alpha)} \frac{\mu_{g}^{1 / 2}\left(\rho D v_{\mathrm{sp}}^{5}\right)^{1 / 6}}{\sigma^{2 / 3}}
$$

where $\mu_{g}$ is the viscosity of air, $v_{\text {sp }}$ is the droplet impact velocity at the onset of splashing, and $\alpha$ is the wedge angle (at the moment of splashing). Past works have found that $\alpha$, at the moment of splashing, remains constant at $\approx 60^{\circ}$, obtaining a value of $\beta \approx 0.11-0.14$, [21]. Our hypothesis is that splashing over hydrophobic and superhydrophobic smooth substrates can be described as a function of $\beta$ and the maximum advancing angle $\theta_{\max }$.

Figure 6 shows our results when parametrized by $\beta$ in terms of $\theta_{\max }$, here, $\alpha$ has been taken as $60^{\circ}$, and the impact speed $U$ has replaced $v_{\text {sp. }}$. As seen in Fig. 6, the splashing behavior is effectively divided for all the different liquids impacting on to all the different solids. Moreover, for 


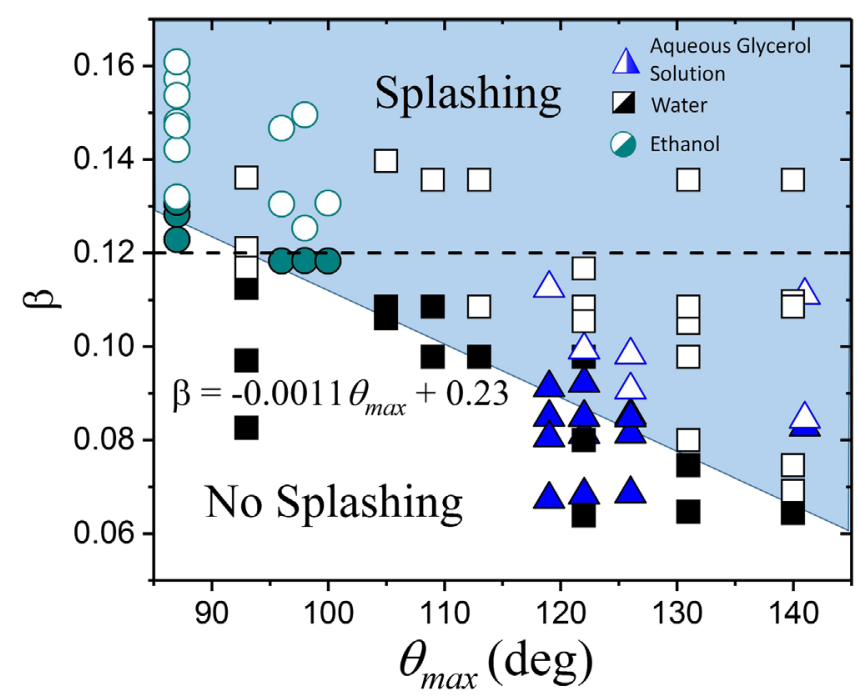

FIG. 6. Splashing in terms of $\theta_{\max }$ and $\beta$. Open symbols represent splashing while solid ones stand for no splashing. The dashed line, $\beta=0.12$, corresponds to the critical splashing value found by de Goede et al. in 2017 [21].

wettable substrates $\left(\theta_{\max }<103\right)$, the data are in agreement with past experiments and simulations (dashed line in Fig. 6) $[7,21]$. The deviation from the current model (dashed line) only occurs when $\theta_{\max }>105$, where the splashing dependency on wettability becomes apparent for hydrophobic substrates. Our premise is that other variables (not explored in this Letter but known to affect splashing), such as ambient pressure or surface roughness also influence the dynamic contact angle. At the timescales of the onset of splashing, our data are in the $\mathrm{Ca}\left(u_{\mathrm{CL}}\right)<0.23$ range, consequently, we assume the contact angle is sufficient to parametrize this system [25].

In this Letter, we have shown that splashing depends on the substrate wettability, through the maximum advancing contact angle $\theta_{\max }$, and the liquid properties for impacting drops. We found that, within our experimental range, $\theta_{\max }$ is greater than $87^{\circ} \pm 4^{\circ}$ for all liquid substrates, and that includes substrates traditionally classified as hydrophilic. Our results shows that the splashing behavior can be parametrized by the maximum advancing contact angle and the splashing ratio. These findings have strong repercussions in several industrial environments, such as in liquid dispensing, liquid coating, sprays, drug delivery, and any other application where splashing can affect coating performance or compromise surface finish or quality.

The research presented in this Letter was financially supported by the Royal Society (Grants No. URF $\backslash R \backslash 180016$ and No. RGF $\backslash E A \backslash 18002$ ), the EPSRC (Grant No. EP/P024173/1), the Mexican Energy Ministry (SENER), CONACyT Mexico, and the Welsh European Funding Office (WEFO) through the Flexible Integrated Energy Systems program (FLEXIS). We thank Corinne Stone from DSTL-UK for providing us the hydrophobic samples.
[1] A. L. Yarin, Annu. Rev. Fluid Mech. 38, 159 (2006).

[2] C. Josserand and S. T. Thoroddsen, Annu. Rev. Fluid Mech. 48, 365 (2016).

[3] P. Tsai, M. H. Hendrix, R. R. Dijkstra, L. Shui, and D. Lohse, Soft Matter 7, 11325 (2011).

[4] C. J. Howland, A. Antkowiak, J. R. Castrejón-Pita, S. D. Howison, J. M. Oliver, R. W. Style, and A. A. CastrejónPita, Phys. Rev. Lett. 117, 184502 (2016).

[5] D. Quéré, Nature (London) 435, 1168 (2005).

[6] J. C. Bird, S. S. Tsai, and H. A. Stone, New J. Phys. 11, 063017 (2009).

[7] G. Riboux and J. M. Gordillo, Phys. Rev. Lett. 113, 024507 (2014).

[8] M. M. Driscoll and S. R. Nagel, Phys. Rev. Lett. 107, 154502 (2011).

[9] A. Latka, A. Strandburg-Peshkin, M. M. Driscoll, C. S. Stevens, and S. R. Nagel, Phys. Rev. Lett. 109, 054501 (2012).

[10] C. S. Stevens, Europhys. Lett. 106, 24001 (2014).

[11] I. V. Roisman, A. Lembach, and C. Tropea, Adv. Colloid Interface Sci. 222, 615 (2015).

[12] R. L. Vander Wal, G. M. Berger, and S. D. Mozes, Exp. Fluids 40, 53 (2006).

[13] J. Palacios, J. Hernández, P. Gómez, C. Zanzi, and J. López, Exp. Therm. Fluid. Sci. 44, 571 (2013).

[14] H. Almohammadi and A. Amirfazli, Soft Matter 13, 2040 (2017).

[15] J. M. Kolinski, L. Mahadevan, and S. M. Rubinstein, Phys. Rev. Lett. 112, 134501 (2014).

[16] A. Latka, Soft Matter 13, 740 (2017).

[17] S. Mandre and M. P. Brenner, J. Fluid Mech. 690, 148 (2012).

[18] J. E. Sprittles, Phys. Rev. Lett. 118, 114502 (2017).

[19] L. Xu, W. W. Zhang, and S. R. Nagel, Phys. Rev. Lett. 94, 184505 (2005).

[20] Z. Jian, C. Josserand, S. Popinet, P. Ray, and S. Zaleski, J. Fluid Mech. 835, 1065 (2018).

[21] T. C. de Goede, N. Laan, K. de Bruin, and D. Bonn, Langmuir 34, 5163 (2018).

[22] Y. Liu, P. Tan, and L. Xu, Proc. Natl. Acad. Sci. U.S.A. 112, 3280 (2015).

[23] S. T. Thoroddsen, K. Takehara, and T. G. Etoh, Phys. Fluids 22, 051701 (2010).

[24] C. Mundo, M. Sommerfeld, and C. Tropea, Int. J. Multiphase Flow 21, 151 (1995).

[25] J. H. Snoeijer and B. Andreotti, Annu. Rev. Fluid Mech. 45, 269 (2013).

[26] C. Antonini, A. Amirfazli, and M. Marengo, Phys. Fluids 24, 102104 (2012).

[27] A. Latka, A. M. P. Boelens, S. R. Nagel, and J. J. de Pablo, Phys. Fluids 30, 022105 (2018).

[28] C. Duez, C. Ybert, C. Clanet, and L. Bocquet, Nat. Phys. 3, 180 (2007).

[29] S. Douezan and F. Brochard-Wyart, Langmuir 27, 9955 (2011).

[30] D. G. Aboud and A.-M. Kietzig, Langmuir 31, 10100 (2015).

[31] K. Yokoi, D. Vadillo, J. Hinch, and I. Hutchings, Phys. Fluids 21, 072102 (2009).

[32] K. Yokoi, Soft Matter 7, 5120 (2011). 
[33] S. T. Thoroddsen, K. Takehara, and T. Etoh, J. Fluid Mech. 706, 560 (2012).

[34] See Supplemental Material at http://link.aps.org/ supplemental/10.1103/PhysRevLett.122.228001 for liquid and substrate properties.
[35] J. B. Lee, D. Derome, R. Guyer, and J. Carmeliet, Langmuir 32, 1299 (2016).

[36] D. Vadillo, A. Soucemarianadin, C. Delattre, and D. Roux, Phys. Fluids 21, 122002 (2009).

[37] I. S. Bayer and C. M. Megaridis, J. Fluid Mech. 558, 415 (2006). 